\title{
OPTIMISTIC DECISION MAKING USING AN APPROXIMATE GRAPHICAL MODEL
}

\author{
BOUTOUHAMI Khaoula and KHELLAF Faiza \\ RIIMA, University of Sciences and Technology Houari Boumediene., Algeria
}

\begin{abstract}
Min-based qualitative possibilistic networks are one of the effective tools for a compact representation of decision problems under uncertainty. The exact approaches for computing decision based on possibilistic networks are limited by the size of the possibility distributions. Generally, these approaches are based on possibilistic propagation algorithms. An important step in the computation of the decision is the transformation of the DAG (Direct Acyclic Graph) into a secondary structure, known as the junction trees $(J T)$. This transformation is known to be costly and represents a difficult problem. We propose in this paper a new approximate approach for the computation of decision under uncertainty within possibilistic networks. The computing of the optimal optimistic decision no longer goes through the junction tree construction step. Instead, it is performed by calculating the degree of normalization in the moral graph resulting from the merging of the possibilistic network codifying knowledge of the agent and that codifying its preferences.
\end{abstract}

\section{KEYWORDS}

Possibilistic decision theory, Min-based possibilistic networks, Moral graph, Data fusion, optimistic criteria.

\section{INTRODUCTION}

More and more, problems of decision making under uncertainty [1] [5]take an important place in Artificial Intelligence (AI) applications. Several decision making tools [2][20]have been developed to assist decision makers in their tasks: simulation techniques, dynamic programming[21], logical decision models[7] and graphical decision models (e.g [4] [12][13] [19]).

Several non-classical theories of uncertainty have been proposed in order to deal with uncertain and imprecise data such as evidence theory [17], Spohn's ordinal conditional functions and possibility theory [24] issued from fuzzy sets theory [23].

This paper focuses on graphical decision models which provide efficient decision tools by allowing a compact representation of decision problems under uncertainty [19]. A decision problem is a choice between a list of possible alternatives taking into account the knowledge of an agent (knowledge is sometimes tainted with uncertainties) as well as his/her preferences. The results of his/her decision are expressed by a set of utilities.

The qualitative possibilistic decision model allows a progressive expression of preferences as well as knowledge of the decision-maker. This model offers two qualitative criteria of utilities for

DOI : 10.5121/ijaia.2015.6201 
the approach of decision under uncertainty: the pessimistic decision criterion and the optimistic decision criterion. Interest in the issue of the calculation of qualitative decision continues to grow and many approaches and models have been proposed [11][16].

In addition to the calculation of decision, the aim of this method is to improve other methods and overcome their limits as regards the presentation form, the calculation time as well as the ease of understanding. In our work we focus on graphical decision models that provide effective tools for decision problems under uncertainty using a compact representation. Several evaluation methods have been proposed to select the optimal decision. Among these methods, there is an exact approach based on possibilistic networks spread. This approach requires a transformation of an original graph into a secondary structure called the junction tree [15] which is used then in various calculations. In this work, our goal is to propose a new approximate approach to compute the optimal optimistic decision. Our approach is based on the moral graph associated with the result of merging the networks representing the agent's beliefs and preferences. This approach has a polynomial complexity [2]. Indeed, it avoids the transformation of the initial graph into a junction tree which is known to be an intractable problem (NP-hard). Using the approximate approach provides very close answers to the exact marginal distributions [2].

The reminder of the paper is organized as follows. The next section briefly recalls the fundamental concepts of possibility theory and min-based possibilistic networks. The main results of merging min-based possibilistic networks are also briefly presented in this section. Section 3 describes the new approach and its use in calculating the optimal optimistic decision and section 4 concludes the paper.

\section{BACKGROUND}

\subsection{Basic concepts of possibility theory}

Probability theory is the fundamental uncertainty theory used in classical decision theory. Despite its fame, probability theory presents some limits since it cannot model qualitative uncertainty and total ignorance is represented by equiprobability which formalizes randomness rather than ignorance. In order to avoid limits of probability theory, non-classical uncertainty theories have been developed. Possibility theory was initially proposed by Zadeh [24] and was developed by Dubois and Prade [8]. This theory offers a suitable framework to handle uncertainty since it allows the representation of qualitative uncertainty.

This section gives a brief refresher on possibility theory which is issued from fuzzy sets theory [24], [18] and represents a main approach in dealing with uncertainty. Let $\mathcal{V}=\left\{\mathrm{A}_{1}, \mathrm{~A}_{2}, \ldots, \mathrm{A}_{\mathrm{n}}\right\}$ be a set of variables. We denote by $D_{A}=\left\{a_{1}, \ldots, a_{n}\right\}$ thedomain associated with the variable $\mathrm{Aa}_{i}$ denotes any instance ofA. The universe of discourse is denoted by $\Omega=\times \times_{A_{i \in V}} D_{A}$, which is the Cartesian product of all variable domains in $\mathcal{V}$. Each element $\omega \in \Omega$ is calledan interpretation which represents a possible state of the world. It is denotedby $\omega=\left(a_{1}, \ldots, a_{n}\right)$ or $\omega=$ $\left(a_{1} \wedge a, \ldots \wedge a_{n}\right)$. Where $\left\{\mathrm{a}_{\mathrm{i}} \mid 1 \leq \mathrm{i} \leq \mathrm{n}\right\}$ are the instancesof the variable $\mathrm{A}_{\mathrm{i}} \cdot \phi, \psi$ denote propositional formulas (corresponding toevents, i.e., subsets of $\Omega$ ) constituted from the variables inv.

\subsubsection{Possibility distribution}

A possibility distribution describes knowledge about the unknown value taken by one or several attributes used to describe states of affairs. For instance it may refer to the age of a man, the size of a building, the temperature of a room, etc. Here it will refer to the ill-known consequence of a 
decision. A possibility distribution can represent a state of knowledge (about the state of affairs) distinguishing what is plausible from what is less plausible, what is the normal course of things from what is not, what is surprising from what is expected.

The possibility distribution is denoted by $\pi$ and it is a mapping from the universe of discourse $\Omega$ to a bounded linearly ordered scale L exemplified by the unit interval $[0,1]$, i.e. $\pi: \Omega \rightarrow$ $[0,1]$.

The particularity of the possibilistic scale $L$ is that it can be interpreted in twofold: in an ordinal manner, i.e. when the possibility degree reflects only an ordering between the possible values and in a numerical interpretation, i.e. when possibility distributions are related to upper bounds of imprecise probability distributions.

This distribution encodes available knowledge on real world: $\pi(\omega)=1$ means that $\omega$ is completely possible and $\pi(\omega)=0$ means that it is impossible to $\omega$ to be the real world. A possibility distribution $\pi$ is said to be $\alpha$-normlized, if its normalization degree $h(\pi)$ is equal to $\alpha$, namely:

$$
h(\pi)=\max \pi(\omega)=\alpha
$$

If $\alpha=1$, then $\phi$ is said to be normalized.

\subsubsection{Possibility and necessity measures}

In probability theory, the quantity $P(\neg \phi)$ is fully determined by $P(\phi)$ since $P(\phi)=1-$ $P(\neg \phi)$.Hence, if $\phi$ isnotprobable, then $\neg \phi$ is necessarily probable. However, the expression "it is not possible that $\phi$ is true" not only implies that " $\neg \phi$ is possible" but it also leads to a stronger conclusion i.e. "it is necessary that $\neg \phi$ ".

Moreover, the expression "it is possible that $\phi$ is true" does not entail anything about the possibility nor the impossibility of $\phi$. Thus, the description of uncertainty about the occurrence of $\phi$ needs two dual measures: the possibility measure $\Pi(\phi)$ and the necessity measure $N(\phi)=1-$ $\Pi(\neg \phi)$ due to the weak relationship existing between these two quantities.

- Possibility measure: this measure evaluates to what extent $\phi$ is consistent with our knowledge. It is given by:

$$
\Pi(\phi)=\{\pi(\omega): \omega \in \phi\}(2)
$$

$\Pi(\phi)$ is called the possibility degree of $\phi$,it corresponds to the possibility to have one of the models of $\psi$ as the real world[21].The degree of possibility satisfies the following properties:

$\Pi(\perp)=0$.

$\Pi(\mathrm{T})=1$.

$\Pi(\phi)=0 . \phi$ is impossible.

$\Pi(\phi)=1$. $\phi$ is completely possible.

$\Pi(\phi)=\Pi(\neg \phi)=1$. $\Phi$ and $\neg \phi$ are totally possible (case of total ignorance).

$\max (\Pi(\phi), \Pi(\neg \phi))=1$. Фor $\neg \phi$ must be possible(criterion of normalisation).

- Necessity measure: it is the dual of the possibility measure. The necessity measure evaluates at which level $\phi$ iscertainly implied by our knowledge. It is given by:

$$
\mathrm{N}(\phi)=1-\Pi(\neg \phi)(3)
$$


Necessity measure corresponds to the certainty degree to have one of the models of $\phi$ as the real world. This measure evaluates at which level $\phi$ is certainly implied by our knowledge represented by $\pi$.

The dual relationship between Пand $\mathrm{N}$ expresses the fact that more is less certain: becomes consistent with available beliefs. The degree of necessity has the following properties:

$\mathrm{N}(\phi)=0$ and $\mathrm{N}(\neg \phi)=0$ 申is total ignorance.

$\mathrm{N}(\phi)=1$ and $\mathrm{N}(\neg \phi)=0$ is certainly true.

$\mathrm{N}(\phi) \in] 0,1[$ and $\mathrm{N}(\neg \phi)=0$. $\phi$ is somewhat certain.

$\mathrm{N}(\psi \wedge \phi)=\min (\mathrm{N}(\psi), \mathrm{N}(\phi))$. Conjonction axiom.

$\mathrm{N}(\psi \vee \phi) \geq \max (\mathrm{N}(\psi), \mathrm{N}(\phi))$. Disjunction axiom.

\subsubsection{Possibilistic conditioning}

The possibilistic conditioning consists in the revision of our initial knowledge, encoded by a possibility distribution $\Pi$, after the arrival of a new certain information $\phi \subseteq \Omega$. The initial distribution $\Pi$ is then replaced by another one, denoted $\Pi^{\prime}=\Pi(. \mid \phi)$. The two interpretations of the possibilistic scale (qualitative and quantitative) induce two definitions of possibilistic conditioning [8]. In this paper, we focus on min-based conditioning (qualitative one) defined by:

$$
\Pi(\omega \mid \phi)=\left\{\begin{array}{cc}
1 & \text { if } \pi(\omega)=\Pi(\phi) \text { and } \omega \vDash \phi i \\
\pi(\omega) & \text { if } \pi(\omega)<\Pi(\phi) \text { and } \omega \vDash \phi i(4) \\
0 \text { otherwise }
\end{array}\right.
$$

The distribution of possibilistic conditioning as defined above means that if $\omega$ falsifies, then it becomes completely impossible. Otherwise, we distinguish two cases: If $\omega$ is a bettermodel, then it becomes totally possible otherwise it keeps the same level of ability.

We also use a so-called min-based independence relation, as a non-interactivity relation [23]. This relation is obtained by using the min-based conditioning Equation 4 and it is defined by:

$$
\forall x, y, z \Pi(x \wedge y \mid z)=\min (\Pi(x \mid z), \Pi(y \mid z)) .
$$

\subsection{Min-based possibilistic network}

\subsubsection{Preliminaries}

There are two ways of knowledge representation: a logical representation and a graphical representation. In this paper we are interested to the graphical representation. It is qualitative network. A possibilistic network is an adaptation of the probabilistic (Bayesian) network, in the sense where we use the same graphic structure which is the direct acyclic graph (DAG)

A min-based possibilistic network [14] over a set of variables $\mathrm{V}$ denoted by $\Pi G_{\min }=$ $\left(G, \pi_{\min }\right)$ is characterized by:

- A graphical component: which is represented by a Directed Acyclic Graph (DAG) where nodes correspond to variables and arcs represent dependence relations between variables.

- Numerical components: these components quantify different links in the DAG by using local possibility distributions for each node $\mathrm{A}$ in the context of its parents denoted by $U_{A}$. More precisely:

- For every root node $A\left(U_{A}=\varnothing\right)$, uncertainty is represented by the a priori possibility degree $\pi(a)$, for each instance a $\in \mathrm{D}_{\mathrm{A}}$, such thatmax $\pi(a)=1$. 
- For the rest of the nodes $A\left(U_{A} \neq \varnothing\right)$, uncertainty is represented by the conditional possibility degree $\pi\left(a \mid U_{A}\right)$, for each instance a $\in \mathrm{D}_{\mathrm{A}}$, and $U_{A} \in \mathrm{D}_{\mathrm{A}}$, such that $\max \pi\left(a \mid U_{A}\right)=1$, for any $U_{A}$.

The set of a priori and conditional possibility degrees induces a unique joint possibility distribution defined by:

Definition 1: Let $\Pi G_{\min }=\left(G, \pi_{\mathrm{G}}\right)$ be a min-based possibilistic network. Given the a priori and conditional possibility distribution, the joint distribution denoted by $\pi_{\mathrm{G}}$, is expressed by the following quantitative chain rule:

$$
\pi_{G}\left(A_{1}, \ldots, A_{n}\right)=\min \left(\Pi\left(A_{i} \mid U_{A i}\right)\right)(6)
$$

Example 1:Let the graphic model of a possibilistic network illustrated in figure 1 and table of initial opportunities distributions is given by the following table:

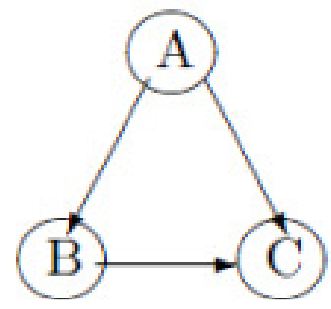

Figure 1. Model of a DAG

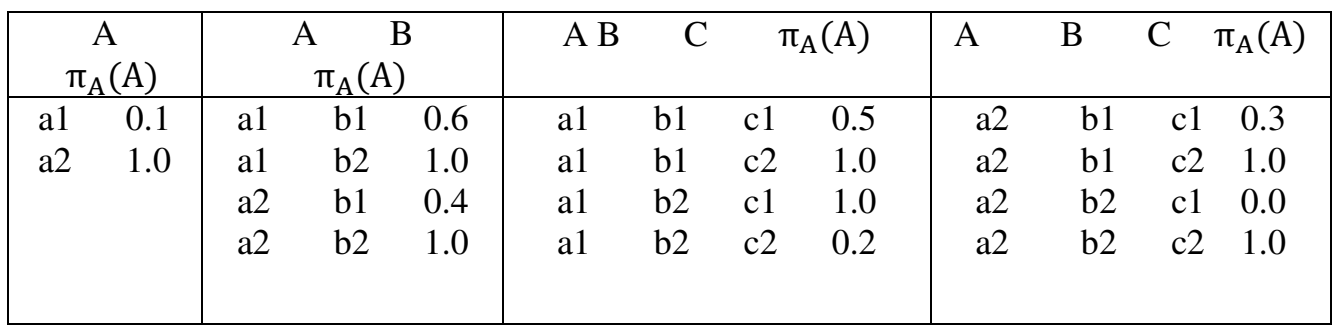

Table 1. Initial possibility distributions

\begin{tabular}{|llll|llll|}
\hline $\mathrm{A}$ & $\mathrm{B}$ & $\mathrm{C}$ & $\pi_{\mathrm{A}}(\mathrm{A})$ & $\mathrm{A}$ & $\mathrm{B}$ & $\mathrm{C}$ & $\pi_{\mathrm{A}}(\mathrm{A})$ \\
\hline $\mathrm{a} 1$ & $\mathrm{~b} 1$ & $\mathrm{c} 1$ & 0.5 & $\mathrm{a} 2$ & $\mathrm{~b} 1$ & $\mathrm{c} 1$ & 0.3 \\
$\mathrm{a} 1$ & $\mathrm{~b} 1$ & $\mathrm{c} 2$ & 1.0 & $\mathrm{a} 2$ & $\mathrm{~b} 1$ & $\mathrm{c} 2$ & 1.0 \\
$\mathrm{a} 1$ & $\mathrm{~b} 2$ & $\mathrm{c} 1$ & 1.0 & $\mathrm{a} 2$ & $\mathrm{~b} 2$ & $\mathrm{c} 1$ & 0.0 \\
$\mathrm{a} 1$ & $\mathrm{~b} 2$ & $\mathrm{c} 2$ & 0.2 & $\mathrm{a} 2$ & $\mathrm{~b} 2$ & $\mathrm{c} 2$ & 1.0 \\
& & & & & & & \\
\end{tabular}

Table 2. Distributions de possibilité jointe

\subsubsection{Fusion of min-based possibilistic networks}

Merging uncertain information [10] is important to exploit complementarities between sources. It provides thus a global and complete point of view. In this paper, we are interested in conjunctive 
mode which makes sense if all sources are considered as equally and fully reliable. One of the basic conjunctive operators is the minimum operation (min).

Given two min-based possibilistic networks $\Pi G_{\min }=\left(G, \pi_{G}\right)$ and $\Pi G^{\prime}{ }_{\min }=\left(G^{\prime}, \pi_{G^{\prime}}\right)$, the result of merging $\Pi G$ and $\Pi G^{\prime}$ is the possibilistic network $\Pi G_{\oplus}=\left(G_{\oplus}, \pi_{\oplus}\right)[22]$, such that:

$$
\forall \omega, \pi_{\oplus}(\omega)=\min \left(\pi_{\mathrm{G}}(\omega), \pi_{\mathrm{G}^{\prime}}(\omega)\right)
$$

The syntactic counterpart of the fusion of two possibility distributions, associated to two possibilistic networks, using the min operator is a new min-based possibilistic network whose definition depends on the union of the two initial ones. In [22], the authors propose two principal classes for merging min-based possibilistic networks:

- Fusion of two possibilistic networks $\Pi G$ and $\Pi G^{\prime}$ having the same network structure. The resulting network $\Pi G_{\oplus}$ retains the same structure: $G_{\oplus}=G=G^{\prime}$. The possibility degrees are computed as follows: for each variable $\mathrm{A}, \pi_{\oplus}\left(A \mid U_{A}\right)=$ $\min \left(\pi_{G}\left(A \mid U_{A}\right), \pi_{G^{\prime}}\left(A \mid U_{A}\right)\right)$.

- Fusion of two possibilistic networks $\Pi G$ and $\Pi G^{\prime}$ withdifferent structures. Two cases are distinguishable:

- The union of graphs is acyclic. In this case, $\Pi G$ and $\Pi G^{\prime}$ are first expanded, with additional variables and links, to $\Pi G 1=\left(G_{\oplus}=G \cup G^{\prime}, \pi_{1}\right)$ and $\Pi G_{1}^{\prime}=(G \oplus$ $\left.=G \cup G, \pi_{1}{ }^{\prime}\right)$ without affecting possibility distribution. The extension process of a min-based possibilistic network can be performed either by adding variables or arcs, namely,

$\checkmark$ Adding variable. The extension of $\Pi G=(\mathrm{G}, \pi)$ by adding a new variable $\mathrm{X} \notin \mathcal{V}$ provides a new min-based possibilistic network $\Pi G_{X}=\left(G_{X}, \pi_{X}\right)$ whichinduces a joint possibility distribution $\pi_{G_{X}}$, such that :

- $\mathrm{GX}=\mathrm{G} \cup\{\mathrm{X}\}$,

- The additional node $X$ will represent the total ignorance, namely $\forall x \in D_{X}, \pi_{X}(x)=1$,

- The remaining variables preserve the same possibility distributions, $\forall Y \in V, Y \neq X, \pi_{X}(Y \mid P \operatorname{ar}(Y))=\pi(Y \mid P \operatorname{ar}(Y))$.

Then, $\forall \omega \in \Omega, \pi_{G}(\omega)=\max \pi_{G_{X}}(x \omega)$.

$\checkmark$ Adding link. The extension of $\Pi G=(\mathrm{G}, \pi)$ by adding a link from $\mathrm{X}$ to $\mathrm{Y}$ $(X \notin \operatorname{Par}(\mathrm{Y}))$ provides a new min-based possibilistic network $\Pi G_{L}=$ $\left(\mathrm{G}_{L}, \pi_{L}\right)$ which induces a joint possibility distribution $\pi \mathrm{GL}$, such that:

$\forall y \in D_{Y}, x \in D_{X}, \operatorname{par}(Y) \in D_{P a r(Y)} \pi_{L}(y \mid \operatorname{par}(Y) x)=\pi(y \mid \operatorname{par}(Y))$. $\forall Z, Z \neq Y, \forall Z \in D Z, \operatorname{par}(Z) \in D P \operatorname{ar}(Z), \pi_{L}(z \mid \operatorname{par}(Z))=\pi(z \mid \operatorname{par}(Z))$. Then, $\forall \omega \in \Omega, \pi_{G}(\omega)=\pi_{G_{L}}(\omega)$.

Since, both initial min-based possibilistic networks have the same structure (by adding nodes and links), then the fusion of same-structure networks is applied.

- The union of graphs is cyclic. In this case, additional variables are added to eliminate cycles. Indeed, the resulting possibilistic network $\Pi G \oplus=(G \oplus, \pi \oplus)$ is obtained by: 
International Journal of Artificial Intelligence \& Applications (IJAIA) Vol. 6, No. 2, March 2015

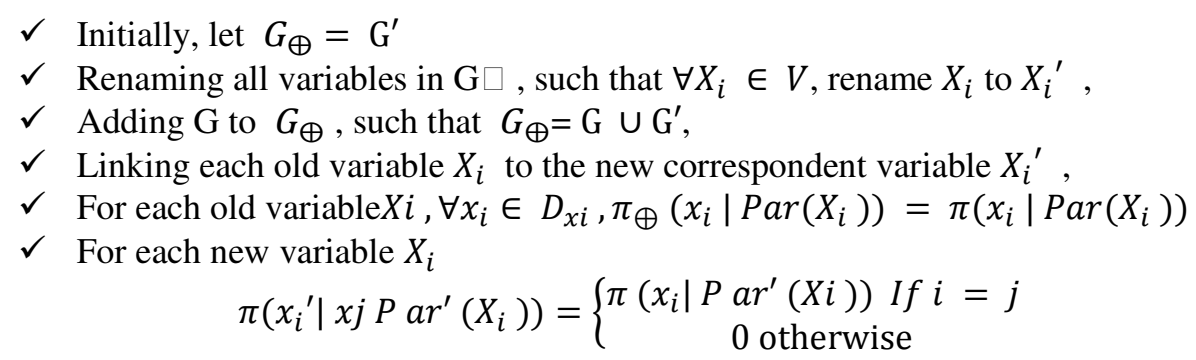

The new conditional distributions relative to the new variables ensure the equivalence between new and old variables.

For more details on the fusion of possibilistic networks see [22].

\section{DECISION MAKING UNDER UNCERTAINTY USING POSSIBILISTIC NETWORKS}

In a problem of decision under uncertainty, knowledge of the decision-maker is generally not very informative. In other words, the agent does not know the real state of the world, but he knows only that this state belongs to a finite set of possible states. A decision system is defined by a finite set of states $S=\left\{s_{1}, s_{2}, \ldots, s_{n}\right\}$, a finite set of consequences $\mathrm{X}$, a set of decisions noted $D=\left\{d_{1}, d_{2}, \ldots, d_{m}\right\}$, and a set of preferences among the consequences. Each decision $d_{i}: S \rightarrow X$ is a function that associates to every possible state of the world a consequence. The preferences among the consequences are encoded by the utility function $v: X \rightarrow U$ where $\mathrm{U}$ is apreferably ordinal scale.

The theory of possibility allows one to express the uncertain knowledge on different states of the world by using a possibility distribution. Indeed, it allows one to represent uncertain knowledge by distinguishing what is plausible to what is less plausible. It provides also a suitable mean to represent preferences on the consequences of decisions in order to distinguish the desirable consequences from the less desirable ones [12].

The uncertainty on the possible states of the world is represented by a normalized possibility distribution $\pi$ thatassociates to a set of state variables a value in the interval $[0,1]$. Likewise, the preferences of the agent are represented by a different possibility distribution $\mu$ that associates to a setof consequences a value in an ordinal scale $U$, represented by the interval [0,1] [12]. We assume that the uncertainties and preferences are commeasurable[9].

In the context of decision theory under uncertainty proposed by Savage, uncertainty of the agent is modeled by a probability distribution $\pi$ on the set of possible states of the world and itspreferences by a utility function $\mu$ with real values on the setX of the possible consequences of his/her actions.

In contrast, in the possibilistic framework, knowledge of the agent is modeled by a normalized possibilistic distribution $\pi$ which is a function from states to a simply ordered scale $L$ of plausibility: for a world $\omega, \pi(\omega) \in L$ : represents thedegree of likelihood that $\omega$ is the real state of the world. If we consider that the information possessed by the agent on the decision problem is purely ordinal, it is reasonable to think that not only his/her knowledge can be expressed by a possibilistic distribution but also his/her preferences [6][16]. A distribution of possibilities can be then seen as a utility [16] function. 
Let $\mu$ be the possibility distribution representing the agent's preferences. $\mu$ takes its values in a simply orderly scale in $[0,1]$. As in Savage theory, an action is represented by a function $d$ that associates to a world an element of X [16].The utility of an action (decision) $d$ in a state $\omega$ and whose consequence is $d(\omega) \in X$ can be evaluated by combiningthe possibility degrees $\pi(\omega)$ and the utilities $\mu(d(\omega))$ in anappropriate manner for all the possible states of world [16].

Two evaluation criteria have been proposed to achieve such combinations assuming some form of commensurability between the scales of plausibility and utility [6][16]:

- Pessimistic criterion (Minimax). Criterion of a pessimistic decision maker: the chosen decision is that having the largest minimum utility :

$$
\mathrm{U}^{*}(\mathrm{~d})=\operatorname{minmax}_{\omega \in \Omega}\left(1-\pi_{\mathrm{Kd}}(\omega), \mu(\omega)\right)
$$

- Optimistic criterion (Maximin): Criterion of an optimistic decision maker: the chosen decision is that having the largest maximum utility :

$$
\mathrm{U}^{*}(\mathrm{~d})=\operatorname{maxmin}_{\omega \in \Omega}\left(\pi_{\mathrm{Kd}}(\omega), \mu(\omega)\right)
$$

In this work, we are interested in the optimistic criterion for the calculation of the decision.

Example 1: Let us consider the problem of deciding whether we should or not take an umbrella, knowing that it would rain. The two min-based possibilistic networks representing knowledge and preferences of the agent are denoted $\Pi \boldsymbol{K}_{\min }$ and $\boldsymbol{\Pi} \boldsymbol{P}_{\min }$ respectively. Before presenting the possibilistic graphs, let us first present the set of nodes used in the networks and their meanings.

- R: It's raining.

- W: The grass is wet.

- UM: Take the umbrella.

- C: Cloudy atmosphere.

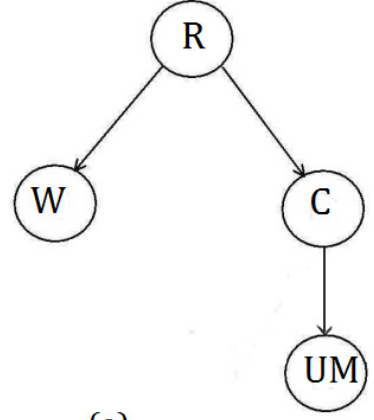

(a)

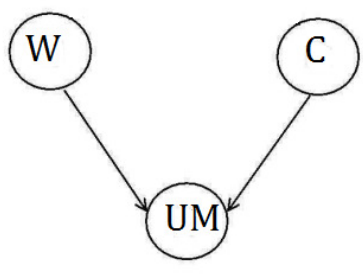

(b)

Figure 2. The possibilistic networks of knowledge and preference of an agent

- Agent's knowledge: described by the min-based possibilistic network $\Pi K_{\min }=$ $\left(G_{K}, \pi_{K}\right)$, where the graphical component GK is given by Figure 2 (a). It contains one possible states of the world $R$, one decision variable $U M$ and two consequences $\{\mathrm{W}, \mathrm{C}\}$. The initial possibility distributions associated with $\Pi K_{\min }$ are given byTables 3 and 4 . We suppose that the variables are binary. 
International Journal of Artificial Intelligence \& Applications (IJAIA) Vol. 6, No. 2, March 2015

\begin{tabular}{|ll|}
\hline $\mathrm{R}$ & $\pi_{\mathrm{K}}(\mathrm{R})$ \\
\hline $\mathrm{r} 1$ & 0.9 \\
$\mathrm{r} 2$ & 1.0 \\
\hline
\end{tabular}

Table 3. Initial possibility distributions relative to $\Pi \mathrm{K}_{\mathrm{min}}$

\begin{tabular}{|ccc|cccc|ccc|}
\hline $\mathrm{W}$ & $\mathrm{R}$ & $\pi_{K}(W \mid R)$ & $\mathrm{C}$ & $\mathrm{R}$ & \multicolumn{2}{|c|}{$\pi_{K}(C \mid R)$} & $\mathrm{UM} \mathrm{C}$ & $\pi_{K}(U M \mid C)$ \\
\hline $\mathrm{w} 1$ & $\mathrm{r} 1$ & 0.4 & $\mathrm{c} 1$ & $\mathrm{r} 1$ & 1.0 & $\mathrm{um} 1$ & $\mathrm{c} 1$ & 1.0 \\
$\mathrm{w} 1$ & $\mathrm{r} 2$ & 1.0 & $\mathrm{c} 1$ & $\mathrm{r} 2$ & 0.2 & $\mathrm{um} 1$ & $\mathrm{c} 2$ & 1.0 \\
$\mathrm{w} 2$ & $\mathrm{r} 1$ & 1.0 & $\mathrm{c} 2$ & $\mathrm{r} 1$ & 0.3 & $\mathrm{um} 2$ & $\mathrm{c} 1$ & 1.0 \\
$\mathrm{w} 2$ & $\mathrm{r} 2$ & 0.0 & $\mathrm{c} 2$ & $\mathrm{r} 2$ & 1.0 & $\mathrm{um} 2$ & $\mathrm{c} 2$ & 1.0 \\
& & & bio1 & of2 & 0.8 & & & \\
& & & bio2 & of1 & 0 & & & \\
\hline
\end{tabular}

Table 4. Initial possibility distributions relative to $\Pi \mathrm{K}_{\min }$

- Agent's preferences: expressed by the min-based possibilistic network $\Pi \mathrm{P}_{\min }=$ $\left(G_{P}, \mu\right)$, where the graphical component $G_{P}$, is given by Figure $2(b)$. It contains one decision variable $U M$ and two consequences $\{\mathrm{W}, \mathrm{C}\}$. The initial possibility distributions associated with $\Pi P_{\text {min }}$ are given by Tables 5 and6.

\begin{tabular}{|cc|cc|}
\hline $\mathrm{W}$ & $\mu(\mathrm{W})$ & $\mathrm{UM}$ & $\mu(\mathrm{UM})$ \\
\hline w1 & 1.0 & um1 & 1.0 \\
w2 & 1.0 & um2 & 1.0 \\
\hline
\end{tabular}

Table5. Initial possibility distributions relative to $\Pi \mathrm{P}_{\min }$

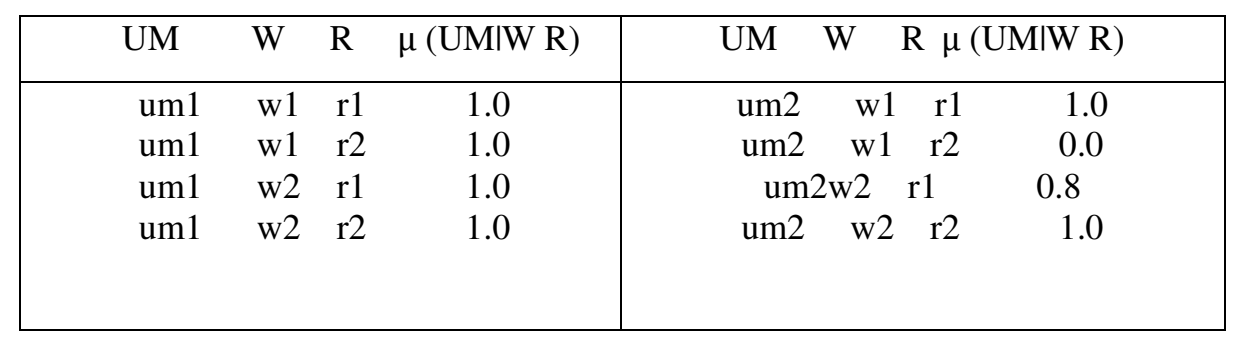

Table 6: Initial possibility distributions relative to $\mathrm{P}_{\min }$

\section{ON THE COMPUTATION OF OPTIMAL OPTIMISTIC DECISIONS BASED ON MIN-BASED FUSION}

Given our graphical model for representing decision making under uncertainty, in this section, we propose an algorithm for dealing with decisions evaluation. In fact, at the semantic level qualitative possibilistic decisions can be viewed as a data fusion problem of two particular possibility distributions: one representing agent's beliefs and the second representing the qualitative utility.

We recall that agent's knowledge and preferences are both represented by two separated minbased possibilistic networks. The first represents agent's beliefs and the second represents the 
qualitative utility. Knowledge and preferences of the agent are both represented by two separated min-based possibilistic networks, namely $\Pi K_{\min }=\left(G_{K}, \pi_{K}\right)$ and $\Pi P_{\min }=\left(G_{P}, \mu\right)$, respectively.

In what follows, we propose a method for computing optimal optimistic decisions based on the fusion of $\pi_{K}$ and $\mu$ (or $\Pi K_{\min }$ and $\Pi P_{\min }$ ). Before describing a fusion process for computing optimal optimistic decisions, it should be noted that each set of decision d induces a possibility distribution $\pi_{K d}$ in the following way [10]:

$$
\pi_{\mathrm{k}_{\mathrm{d}}}(\omega)=\min \left(\pi_{\mathrm{k}}(\omega), \pi_{\mathrm{d}}(\omega)\right)(10)
$$

\subsection{Describing optimistic decisions as a fusion process}

We recall that making a decision comes down to choosing a subset $\mathrm{d}$ of the decision set $\mathrm{D}$ which maximizes the optimistic qualitative utility by:

Where,

$$
\mathrm{U}^{*}(\mathrm{~d})=\operatorname{maxmin}_{\omega \in \Omega}\left(\pi_{\mathrm{k}_{\mathrm{d}}}(\omega), \mu(\omega)\right)(11)
$$

$$
\pi_{d}(\omega)=\left\{\begin{array}{cc}
1 & \text { if } \omega \vDash \phi i \\
0 & \text { otherwise }
\end{array}(12)\right.
$$

Using equation (12), the optimistic utility decision $U^{*}(d)$ becomes:

$$
\mathrm{U}^{*}(\mathrm{~d})=\operatorname{maxmin}_{\omega \in \Omega}\left(\min \left(\pi_{\mathrm{K}}(\omega), \mu(\omega)\right), \pi_{\mathrm{d}}(\omega)\right)
$$

Using technical merging of two min-based possibilistic networks, this Equation (13) down to:

where $\pi_{\oplus}(\omega)=\min \left(\pi_{K}(\omega), \mu(\omega)\right)$.

$$
\mathrm{U}^{*}(\mathrm{~d})=\operatorname{maxmin}_{\omega \in \Omega}\left(\min \left(\pi_{\oplus}(\omega), \pi_{\mathrm{d}}(\omega)\right)(14)\right.
$$

Example 2: The two DAGs $\left(G_{K}\right.$ and $\left.G_{P}\right)$ given in Example 1, Figure 1 havea different structures. Their union is acycles, the result of merging $\Pi K_{\min }=\left(G_{K}, \pi_{K}\right)$ and $\Pi P_{\min }=\left(G_{P}, \mu\right)$,is the min-based possibilistic network $\Pi G_{\oplus}=\left(G_{\oplus}, \pi_{\oplus}\right)$ where $G_{\oplus}$, isgiven in Figure $3 \mathrm{G}_{\oplus}$ is simply the union of the two graphs of Figure 1 and 2. The resulted min-based possibilistic network $\Pi G_{\oplus}$ induces a unique possibility distribution $\pi_{\oplus}$.

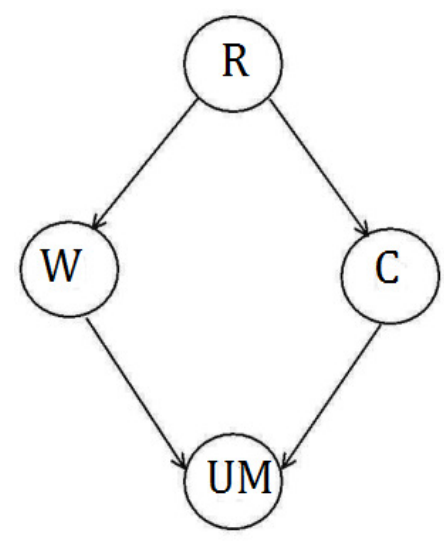

Figure3. The $\mathrm{DAG} \mathrm{G}_{\oplus}$ 
The initial possibility distributions are given by Tables 7 and 8 .

\begin{tabular}{|c|c|c|c|c|c|c|}
\hline & $\pi_{K}(\mathrm{R})$ & W & $\mathrm{R}$ & $\pi_{K}(W \mid R)$ & C $\mathrm{R}$ & $\pi_{\mathrm{K}}(\mathrm{C} \mid \mathrm{R})$ \\
\hline r1 & 0.9 & w1 & r1 & 0.4 & $\mathrm{c} 1$ & 1.0 \\
\hline \multirow[t]{3}{*}{ r2 } & 1.0 & w1 & r2 & 1.0 & $\mathrm{c} 1$ & 0.2 \\
\hline & & w2 & r1 & 1.0 & $c 2$ & 0.3 \\
\hline & & w2 & r2 & 0.0 & $\mathrm{c} 2$ & 1.0 \\
\hline
\end{tabular}

Table 7. Initial possibility distributions relative to $\Pi_{\oplus}$

\begin{tabular}{|c|c|c|c|c|c|}
\hline UMV & \multicolumn{2}{|c|}{ C $\mu(\mathrm{UM} \mid \mathrm{W}$ C) } & UM & \multicolumn{2}{|c|}{$\mathrm{W} \quad \mathrm{C} \mu(\mathrm{UM} \mid \mathrm{W} \mathrm{C})$} \\
\hline um1 & w1 c1 & 1.0 & um2 & w1 & 1.0 \\
\hline um1 & w1 & 0.0 & um2 & w1 & 1.0 \\
\hline um1 & w2 & 0.8 & um2 & w2 c1 & 1.0 \\
\hline um1 & w2 & 1.0 & um2 & w2 c2 & 1.0 \\
\hline & & & bic & 2 of 1 & 0 \\
\hline & & & bic & of 2 & 1 \\
\hline
\end{tabular}

Table 8.Initial possibility distributions relative toПG $\mathrm{G}_{\oplus}$.

\subsection{Computing optimal decisions using moral graph}

In this section, we present the propagation process based on moral graph to compute optimal decisions. It is composed of several steps, which progressively get close to exact possibility degrees (i.e. converges to exact values). The first step consists in transforming the initial possibilistic graph in to an equivalent undirected graph, called here for simplicity moral graph, where each node (called cluster) contains a variable from the initial graph and its parents. The clusters are quantified by local joint possibility distributions instead of the initial conditional ones. Then, several stability procedures are used in order to guarantee that joint distribution relative to any cluster is in agreement with those of its adjacent clusters.

Computing the optimistic optimal decisions amounts to find the normalization degree of the moral graph resulting from the merging of the two possibilistic networks, the first min-based possibilistic network encodes a joint possibility distribution representing available knowledge and the second one encodes the qualitative utility, without going through the junction tree.Note that the construction of the moral graph is done only once and has a polynomial complexity. However, the simple stabilization procedure, multiple stabilization procedure and initialization (see below) (which are all three polynomials) are repeated for each decisiond*.

\subsubsection{Building the moral graph.}

The first step is to transform the initial network in to an equivalent secondary structure, called moral graph for simplicity of notation, and denoted by $\mathcal{M G}$. Each node in the moral graph MG is called a cluster and it is constructed by adding to each node (variable) from the initial network its parent set. This construction way insures that for any variable A corresponds only one cluster in 
$\mathcal{M} \mathcal{G}$ denoted by $\mathrm{C}_{i}$. Between any two clusters $\mathrm{C}_{i}$ and $\mathrm{C}_{j}$ with a non-empty intersection exits an edge labeled with a separator, denoted by $S_{i j}$, containing the common variables in $C_{i}$ and $C_{j}$.

The construction of the possibilistic moral graph, noted $\mathcal{M G}$, from the initial graph is done as follows:

- $\quad$ For each variable Ai ,form a clusterC $C_{i}=A_{i} \cup U_{A}$

- For each edge connecting two nodes $A_{i}$ and $A_{j}$ : forman undirected edge in the moral graph between thecluster $\mathrm{C}_{i}$ and the cluster $\mathrm{C}_{j}$ labeled with a separator $S_{i j}$ corresponding to their intersection.

Example 3: Let us continue with Example 2. We need to compute the optimal optimistic decision $\mathrm{UM}=\{\mathrm{um} 1$; um2 $\}$. First, we start by constructing the moral graph (see Figure4) associated with the graph $G_{\oplus}$ (Figure 3 ) representing the fusion of $\Pi K_{\min }$ and $\Pi P_{\min }$. The resulted moral graph contains four cluster $\mathrm{C} 1=\{\mathrm{R}\}, \mathrm{C} 2=\{\mathrm{R}, \mathrm{W}\}, \mathrm{C} 3=\{\mathrm{R}, \mathrm{C}\}$ and $\mathrm{C} 4=\{\mathrm{R}, \mathrm{W}, \mathrm{UM}\}$ and their separator $S 12=\{R\}, S 12=\{R\}, S 13=\{R\}, S 23=\{R\}, S 24=\{w\}$ and $S 34=\{c\}$.

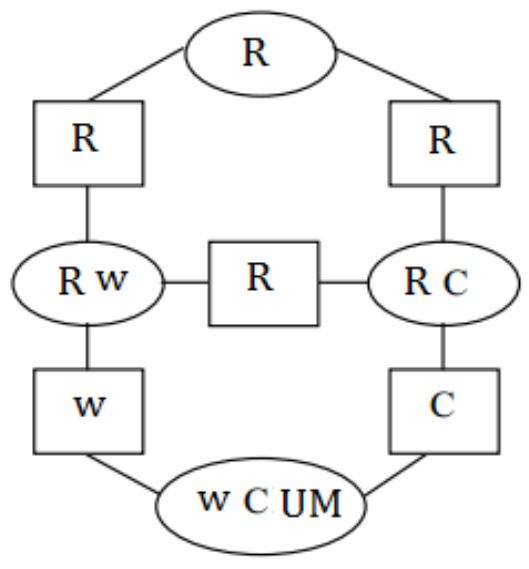

Figure.4. Moral Graph MG of th DAG in Figure 3

\subsubsection{Initialization.}

For a given decision $\mathrm{d}$, once the moral graph is built, the initial conditional distributions are transformed into local joints in order to quantify it. Namely, for each cluster $\mathrm{C}$ of $\mathcal{M} \mathcal{G}$, we assign a local joint distribution relative to its variables, called potential and denoted $\pi_{\mathrm{Ci}}^{\mathrm{I}}$.

The quantification is proceeds by taking into account the decision $d$ as follows:

- $\quad$ For each cluster $\mathrm{C}_{i}$, (resp. $\left.S_{i j}\right) \pi_{\mathrm{Ci}}^{\mathrm{I}} \leftarrow 1$. $\left(\operatorname{resp} . S_{i j} \leftarrow 1\right)$

- For each variable $A_{i} \quad, \quad$ choose a cluster $\mathrm{C}_{i}$ containing $A_{i} \cup U_{A} \pi_{C_{i}} \leftarrow$ $\min \left(\pi_{C_{i}}, \pi_{\oplus}\left(A_{i} \mid U_{A}\right)\right)$.

- $\quad$ Encode the evidence $D=d_{i}$ as likelihood $\Lambda_{D}(d)$ :

$$
\Lambda_{D}(d): \begin{cases}1 & D \text { is instanciated as } d \\ 0 & D \text { is instanciated as a value } d^{\prime} \neq d\end{cases}
$$

- Identify a cluster $C_{i}$ containing D: 


$$
\pi_{\mathrm{Ci}}^{\mathrm{I}} \leftarrow \min \left(\pi_{\mathrm{Ci}}^{\mathrm{I}}, \Lambda_{D}\right)
$$

It is clear that the joint distribution encoded by the initialized moral graph is equivalent to the one encoded by the initial networks since the local joint distributions in clusters are equal to the initial local conditional distributions.

Note that Equations 16 and 17 do not appear in standard initialization of moral graph associated with standard min-based possibilistic networks. It is proper to possibilistic decision problem. By entering the fact $\mathrm{D}=\mathrm{d}$, the moral graph $\mathcal{M} \mathcal{G}$ encodes $\pi$

$$
\pi_{\mathcal{M G}}(\omega)=\min \left(\pi_{\oplus}(\omega), \pi_{d}(\omega)\right)
$$

where $\pi_{\mathcal{M G}}$ can be redefined from $\mathcal{M} \mathcal{G}$ as follows:

Definition 1: The joint distribution associated with the moral graph $\mathcal{M} \mathcal{G}$ is expressed by:

$$
\pi_{\mathcal{M C}}=\min _{\mathrm{i}: 1, \mathrm{~m}} \pi_{C_{i}}
$$

where $\mathrm{m}$ is the number of clusters in $\mathcal{M} \mathcal{G}$.

Then the qualitative utility associated with a decision $d$ is summarized by the following proposition:

Proposition 1: Let $\Pi K_{\min }=\left(G_{K}, \pi_{K}\right)$, be a min-based possibilistic network representing agent's beliefs and $\Pi P_{\min }=\left(G_{P}, \mu\right)$ be a min-based possibilistic network representing agent's preferences. Let $\Pi G_{\oplus}=\left(G_{\oplus}, \pi_{\oplus}\right)$ be the result of merging $\Pi K_{\min }$ and $\Pi P_{\min }$ using the min operator. Let $\mathcal{M} \mathcal{G}$, be the moral graph corresponding to $\Pi G_{\oplus}$ generated using the above initialization procedure. Then,

$$
\mathrm{U}^{*}(\mathrm{~d})=\max _{\omega \in \Omega}(\mathcal{M} \mathcal{G}(\omega))
$$

Where $U^{*}(d)$ is given in Equation 14 .

Hence, after the initialization step, the moral graph really encodes the possibilistic optimistic decision. The next step is used to apply the simple stabilization procedure and multiple stabilization procedure in order to efficiently determine the value of $U^{*}(d)$.

\subsubsection{Simple Stability Procedure.}

The simple stabilization procedure ensures that the potential of each clique is in agreement with that of its neighbors. This procedure is applied through a mechanism of passage of messages between different cliques. Indeed, each separator collects information from its corresponding cliques in order to distribute it then to each of them in order to update them.

The potentials of any adjacent clusters $C_{i}$ and $C_{j}$ (with separator $S_{i j}$ ) are updated as follows:

- Collect evidence(Update separator) :

$$
S_{i j}^{t+1} \leftarrow \min \left(\max _{\mathrm{Ci} / \mathrm{Sij}} \pi_{\mathrm{Ci}}^{t}, \max _{\mathrm{Cj} / \mathrm{Sij}} \pi_{\mathrm{Ci}}^{\mathrm{t}}\right)
$$

- Distribute evidence (Update clusters) : 
International Journal of Artificial Intelligence \& Applications (IJAIA) Vol. 6, No. 2, March 2015

$$
\begin{aligned}
& \pi_{C i}^{t+1} \leftarrow \min \left(\pi_{\mathrm{Ci}}^{t}, \pi_{\mathrm{Sij}}^{t+1}\right) \\
& \pi_{C j}^{t+1} \leftarrow \min \left(\pi_{\mathrm{Cj}}^{t}, \pi_{\mathrm{Sij}}^{t+1}\right)
\end{aligned}
$$

These two steps are repeated until reaching the stability of all clusters. This procedure is defined as follows:

Definition 2: Let $\mathrm{C}_{i}$ and $\mathrm{C}_{j}$ be two adjacent clusters in a moral graph $\mathcal{M} \mathcal{G}$, and let $\mathrm{S}_{i j}$ be their separator. The separator $\mathrm{S}_{i j}$ is said to be stable if:

$$
\max _{\mathrm{Ci} / \mathrm{Sij}} \pi_{\mathrm{Ci}}^{\mathrm{I}}=\max _{\mathrm{Cj} / \mathrm{Sij}} \pi_{\mathrm{Ci}}^{\mathrm{I}}
$$

Where $\max _{\mathrm{Ci} / \mathrm{Sij}} \pi_{\mathrm{C}_{\mathrm{i}}}$ is the marginal distribution of $S_{i j}$ defined from $\pi_{\mathrm{Ci}}^{\mathrm{I}}$ (resp. $\pi_{\mathrm{Cj}}^{\mathrm{I}}$ ).

A moral graph $\mathcal{M G}$ is stable if all its separators are stable.

Proposition 2: Let $\mathcal{M G}$ be a stabilized moral graph, let $\pi_{\mathcal{M} \mathcal{G}}$ be the joint distribution encoded by MG after the initialization procedure. Then,

$$
\forall \mathrm{C}_{i}, \max \pi_{\mathcal{M} \mathcal{G}}^{S} \geq \alpha
$$

Where, $\alpha$ is the maximum value in all clusters.

Algorithm1: Simple stability procedure

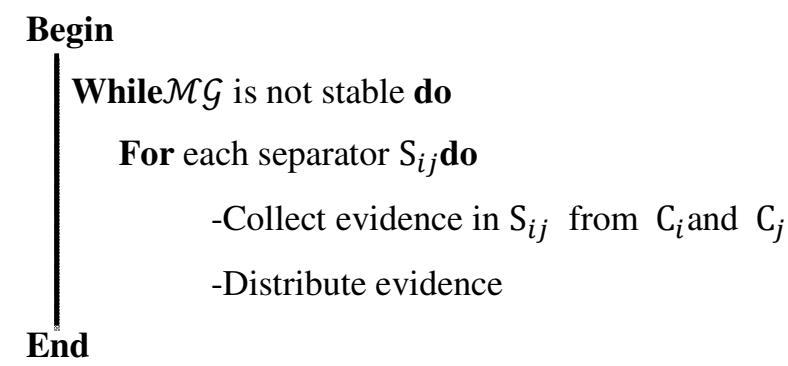

\section{- $\quad$ The theoretical complexity.}

In this procedure, a separator will be treated if one of its corresponding clusters has been modified. Moreover, a cluster will be treated if one of its corresponding separators has been modified. Thus, the moral graph is considered as stable if none of its clusters has been modified. It can be shown that the simplest ability is reached after a finite number of message passes, which can be evaluated as follows:

Let $N$ be the number of clusters, $\mathcal{M}$ bethenumberofseparatorsand $P$ thenumberofvaluesinthe possibilistic scale relative to all the clusters. The iteration in the simplest ability procedure is repeated until there is no modification in the clusters. The maximal number of iterations occurs when a degree is modified in one cluster during one iteration, thus we can have atmost $N *$ $P$ iterations.Each iteration runs $O(\mathcal{M})$ timestheCollet-Distribute evidence. Thus, the theoretical complexity is $O(\mathcal{M} * N * P)$ and hence the stability is a polynomial procedure. 


\subsubsection{Multiple Stability Procedure.}

[3]Proved that the simple stabilization procedure does not always guarantee accurate marginal. One needs to stabilize each clique with respect to all of its adjacent cliques but this can turns out to be very costly in terms of calculation if the number of cliques is important. For that, [3] has proposed to follows several steps in stabilizing the possibilistic moral graph over subsets of its adjacent cliques. Authors of [3] have proposed several progress ivestabilization procedures based on $n$ parents, $n$ children, $n$ parents children and $n$ neighbors by varying the value of $n$ from 2 up to the cardinality of the considered subset. To illustrate the multiple stabilization procedure, we consider the case of two parent's stabilization. The principle of this procedure is to ensure for each clique, with at least two parents, its stabilization over each pair of parents. The updating of any cluster $C_{i}$ with respect to two of its parents $C_{j}$ and $C_{k}$ is performed as follows:

- Compute the potential of $\mathrm{C}_{i}$ using $\mathrm{C}_{j}$ and $\mathrm{C}_{k}$ :

$$
\pi_{C} \leftarrow \min \left(\pi_{\mathrm{Cj}}^{t}, \pi_{\mathrm{Ck}}^{t}\right)(26)
$$

- Distribute the potential of $S$ using $C$ :

$$
\pi_{S} \leftarrow \max _{C_{\backslash S}} \pi_{C}(27)
$$

- Compute the potential of $\mathrm{C}_{i}$ using $\mathrm{C}_{j}$ and $\mathrm{C}_{k}$ :

$$
\pi_{\mathrm{Ci}}^{t+1} \leftarrow \min \left(\pi_{\mathrm{Ci}}^{t}, \pi_{S}\right)
$$

Once stability has been reached, the calculation of qualitative utility over a decision $\mathrm{d}$ will be obtained as follows:

Proposition 3. Let $\Pi K_{\min }=\left(G_{K}, \pi_{K}\right)$ be a min-based possibilistic network representing agent's beliefs and $\Pi P_{\min }=\left(G_{P}, \mu\right)$ a min-based possibilistic network representing agent's preferences. $\Pi G_{\oplus}$ is the result of merging of $\Pi K_{\text {min }}$ and $\Pi P_{\text {min }}$ by using the min operator. Let $\mathcal{M} \mathcal{G}$ be the moral graph of $\Pi G_{\oplus}$. The computation of optimistic decisionsreturns to calculate the normalization degree of $M G$ :

$$
\mathrm{U}^{*}(\mathrm{~d})=\max _{C_{i}}\left(\pi_{C_{i}}\right)
$$

\subsection{Algorithm}

The computation of the optimal optimistic decisions is obtained using the following algorithm.

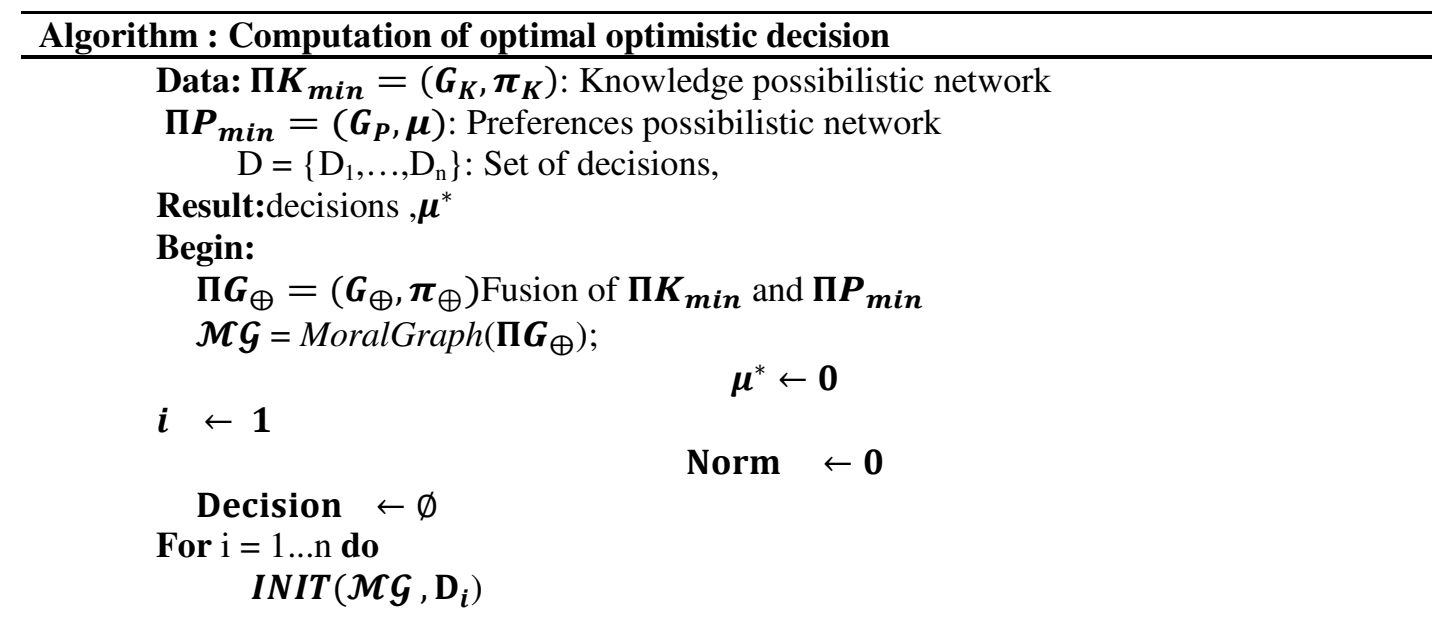




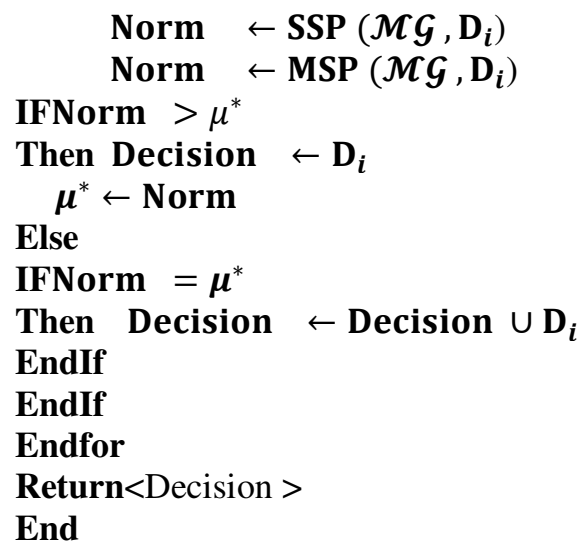

The result of the fusion steps of the two initial min-based networks $\Pi K_{\min }$ and $\Pi P_{\min }$ is the minbased network $\Pi G_{\oplus}=\left(G_{\oplus}, \pi_{\oplus}\right)$. The construction of the moral graph $\mathcal{M} \mathcal{G}$ associated with the resulted fusion min-based network $\Pi G_{\oplus}=\left(G_{\oplus}, \pi_{\oplus}\right)$ is ensured by calling the function moral graph MoralGraph $\left(\boldsymbol{\Pi G _ { \oplus }}\right)$. In addition, the function $\operatorname{Init}(\boldsymbol{M} \mathcal{G}$; d) corresponds to the initialization step. Similarly, the proceduresSSP $\left(\mathcal{M} \mathcal{G}, \mathbf{D}_{\boldsymbol{i}}\right)$ corresponds to the simple stabilization procedure, MSP $\left(\mathcal{M} \mathcal{G}, \mathrm{D}_{i}\right)$ corresponds to the multiple stabilization and returns a normalization degree relative to $\mathcal{M} \mathcal{G}$.

As it was already stated, the construction of the moral graph is only done once but the initialization, the simple stabilization procedure and the multiple stability procedure step sare repeated for each decision. More precisely, for each decision $d_{i}=\left\{d_{1}, \ldots, d_{n}\right\}$ a call to the initialization, the simple stabilization procedure and the multiple stability procedure occurred.

The initialization function $\operatorname{Init}(\boldsymbol{M} \mathcal{G}$; d)allows the parameterization of the moral graph by the decision $\mathrm{d}_{\mathrm{i}}$.

As for the simple stabilization procedureSSP( $\left.\mathcal{M} \mathcal{G}, \mathbf{D}_{\boldsymbol{i}}\right)$ and the multiple stability procedure $\operatorname{MSP}\left(\mathcal{M G}, \mathbf{D}_{\boldsymbol{i}}\right)$, they allows the computation of a normalization degree associated to the parameterized moral graph. Finally, the algorithm returns optimal decisions, those that maximize the normalization degree relative to the moral graph.

The interesting feature of our approach is that the decision calculation process has a polynomial complexity: the complexity of the merge process is polynomial, in particular when the union of the two graphs is free of cycles. In this case, the complexity of the fusion process is linear with respect to the number of variables and parameters of the two graphs, as well as the complexity of two stabilization procedures are polynomial.

Example 4: Let us continue with Example 3. We need to compute the optimal optimistic decision $\mathrm{UM}=\{\mathrm{um} 1 ; \mathrm{um} 2\}$. First, we start by constructing the moral graph (see Figure4) associated with the graph $G_{\oplus}$ (Figure 3 ) representing the fusion of $\Pi K_{\min }$ and $\Pi P_{\min }$. The resulted moral graph contains four cluster $\mathrm{C} 1=\{\mathrm{R}\}, \mathrm{C} 2=\{\mathrm{R}, \mathrm{W}\}, \mathrm{C} 3=\{\mathrm{R}, \mathrm{C}\}$ and $\mathrm{C} 4=\{\mathrm{R}, \mathrm{W}, \mathrm{UM}\}$ and their separator $S 12=\{R\}, S 12=\{R\}, S 13=\{R\}, S 23=\{R\}, S 24=\{w\}$ and $S 34=\{c\}$.

Then, for each decision value in $\mathrm{UM}=\{\mathrm{um} 1 ; \mathrm{um} 2\}$, we must run the algorithm in order to compute the normalization degree associated with the moral graph. 


\begin{tabular}{|cl|cl|}
\hline$\omega$ & $\pi_{\mathcal{M G}}$ & $\omega$ & $\pi_{\mathcal{M C} \mathcal{G}}$ \\
\hline r1 w1 c1 um1 & 0.3 & r2 w1 c1 um1 & 0.0 \\
r1 w1 c1 um2 & 0.3 & r2 w1 c1 um2 & 0.0 \\
r1 w1 c2 um1 & 0.0 & r2 w1 c2 um1 & 0.0 \\
r1 w1 c2 um2 & 1.0 & r2 w1 c2 um2 & 0.0 \\
r1 w2 c1 um1 & 0.3 & r2 w2 c1 um1 & 0.8 \\
r1 w2 c1 um2 & 0.3 & r2 w2 c1 um2 & 0.9 \\
r1 w2 c2 um1 & 0.4 & r2 w2 c2 um1 & 0.2 \\
r1 w2 c2 um2 & 0.4 & r2 w2 c2 um2 & 0.2 \\
\hline
\end{tabular}

\section{Step 1: $\mathbf{U M}=\mathbf{u m 1}$}

Table 9. Joint distribution $\pi_{\mathcal{M G}}$

In this case, the fact that $\mathrm{UM}=\mathrm{um} 1$ is encoded as follows:

$$
\Lambda_{U M}(u m 1): \begin{cases}1 & U M \text { is instanciated as um1 } \\ 0 & \text { UM is instanciated as um2 }\end{cases}
$$

The table 10 represents the joint distribution encoded by $\mathcal{M} \mathcal{G}$ after the initialization procedure us.

\begin{tabular}{|cl|cl|}
\hline$\omega$ & $\pi_{\mathcal{M G}}$ & $\omega$ & $\pi_{\mathcal{M G}}$ \\
\hline r1 w1 c1 um1 & 0.3 & r2 w1 c1 um1 & 0.0 \\
r1 w1 c1 um2 & 0.0 & r2 w1 c1 um2 & 0.0 \\
r1 w1 c2 um1 & 0.0 & r2 w1 c2 um1 & 0.0 \\
r1 w1 c2 um2 & 0.0 & r2 w1 c2 um2 & 0.0 \\
r1 w2 c1 um1 & 0.3 & r2 w2 c1 um1 & 0.8 \\
r1 w2 c1 um2 & 0.0 & r2 w2 c1 um2 & 0.0 \\
r1 w2 c2 um1 & 0.4 & r2 w2 c2 um1 & 0.2 \\
r1 w2 c2 um2 & 0.0 & r2 w2 c2 um2 & 0.0 \\
\hline
\end{tabular}

Table 10. Joint distributions $\pi_{\mathcal{M G}}$ after the initialization procedure

Once the moral graph is quantified, then the simple stabilization procedure allows us to compute the normalization degree of the moral graph which corresponds to the normalization degree of any cluster. Using this procedure, we obtain:

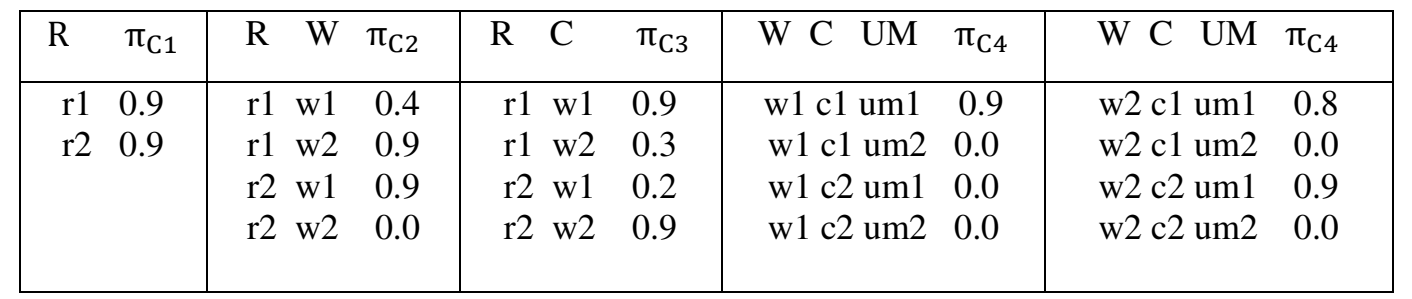

Table 11. Normalized potentials with UM=um1 
$\max _{\pi C 1}=\max _{\pi C 2}=\max _{\pi C 3}=\max _{\pi C 4}=0.9$

From the table 8 we can check that: $\mathrm{h}\left(\pi_{\mathcal{M G}}\right)=0.8 \neq 0.9$, which means that the moral graph is not consistent. So we must to re-stabilize the moral graph using the multiple.stability procedure. Using this procedure, we obtain:

\begin{tabular}{|c|c|c|c|c|c|c|c|c|c|}
\hline $\mathrm{R}$ & $\pi_{\mathrm{C} 1}$ & $\mathrm{R} \quad \mathrm{W}$ & $\pi_{\mathrm{C} 2}$ & $\mathrm{R} \quad \mathrm{C}$ & $\pi_{\mathrm{C} 3}$ & W C UM & $\pi_{C 4}$ & W C UM & $\pi_{\mathrm{C} 4}$ \\
\hline $\mathrm{r} 1$ & 0.8 & r1 w1 & 0.4 & r1 w1 & 0.4 & w1 c1 um1 & 0.3 & w2 c1 um1 & 0.8 \\
\hline \multirow[t]{3}{*}{ r2 } & 0.4 & r1 w2 & 0.3 & r1 w2 & 0.3 & $\mathrm{w} 1 \mathrm{c} 1 \mathrm{um} 2$ & 0.0 & w2 $\mathrm{c} 1 \mathrm{um} 2$ & 0.0 \\
\hline & & & 0.8 & r2 w1 & 0.2 & $\mathrm{w} 1 \mathrm{c} 2 \mathrm{um} 1$ & 0.0 & w2 c2 um1 & 0.4 \\
\hline & & r2 w2 & 0.0 & r2 w2 & 0.8 & $\mathrm{w} 1 \mathrm{c} 2 \mathrm{um} 2$ & 0.0 & w2 c2 um2 & 0.0 \\
\hline
\end{tabular}

Table 12. Normalized potentials with UM=um1

$\max _{\pi C 1}=\max _{\pi C 2}=\max _{\pi C 3}=\max _{\pi C 4}=0.8$

The normalization degree of the moral graph is: $\mathrm{U}^{*}(\mathrm{um} 1)=0.8$

\section{Step 2: $\mathrm{UM}=\mathbf{u m 2}$}

We repeat the same procedure described in the previous step, with:

$$
\Lambda_{U M}(u m 2): \begin{cases}1 & U M \text { is instanciated as um2 } \\ 0 & \text { UM is instanciated as um1 }\end{cases}
$$

The table 13 represents the joint distribution encoded by $\mathcal{M} \mathcal{G}$ after the initialization procedure us.

\begin{tabular}{|cl|cl|}
\hline$\omega$ & $\pi_{\mathcal{M G}}$ & $\omega$ & $\pi_{\mathcal{M G}}$ \\
\hline r1 w1 c1 um1 & 0.0 & r2 w1 c1 um1 & 0.0 \\
r1 w1 c1 um2 & 0.3 & r2 w1 c1 um2 & 0.0 \\
r1 w1 c2 um1 & 0.0 & r2 w1 c2 um1 & 0.0 \\
r1 w1 c2 um2 & 1.0 & r2 w1 c2 um2 & 0.0 \\
r1 w2 c1 um1 & 0.0 & r2 w2 c1 um1 & 0.0 \\
r1 w2 c1 um2 & 0.3 & r2 w2 c1 um2 & 0.9 \\
r1 w2 c2 um1 & 0.0 & r2 w2 c2 um1 & 0.0 \\
r1 w2 c2 um2 & 0.4 & r2 w2 c2 um2 & 0.2 \\
& & & \\
\hline
\end{tabular}

Table 13. Joint distributions $\pi_{\mathcal{M G}}$ after the initialization procedure

In the same way, once the moral graph is quantified, using the simple stabilization procedure and the multiple stabilization procedure, we obtain:

$\mathrm{U}^{*}(\mathrm{um} 2)=\max _{\pi C 1}=\max _{\pi C 2}=\max _{\pi C 3}=\max _{\pi C 4}=1.0$

Thus, we can conclude that the optimal optimistic decision is $\mathbf{U M}=\mathbf{u m} \mathbf{2}$ with the maximal qualitative utility which equals 1.0 
International Journal of Artificial Intelligence \& Applications (IJAIA) Vol. 6, No. 2, March 2015

\section{CONCLUSION}

In this paper, we proposed a new approximate approach for the computation of the qualitative possibilistic optimal optimistic decision in a graphical context. Our approach first merges possibilistic networks associated with available uncertain knowledge and possibilistic networks associated with agent's preferences. We then showed that computing optimistic decisions comes down to computing a normalization degree of the moral graph associated to the result graph of merging agent's beliefs and preferences networks. This allows an efficient computation of optimal decisions.

This approach allows one to avoid the transformation of the initial graph into a junction tree which is known as a difficult problem. This approach is interesting when accurate approaches fail, i.e., when the generation of the local possibility distributions by the standard algorithm is impossible or takes a too long response time. In such case, our approach provides answers that are very close to the exact marginal distributions.

As a future work, we plan to apply our solution to deal with the pessimistic decisions for possibilistic decision problems.

\section{REFERENCES}

[1] Achs, A.: Computed Answer from Uncertain Knowledge: A Model for Handling Uncertain Information. Computers and Artificial Intelligence, Vol. 26, 2007, No. 1,pp. 63-76.

[2] Bélanger, M. Martel, J.M.: Explanations for a Decision Support System based on MCDA. Computing And Informatics, Vol. 25, 2006, No. 2-3, pp. 195-221.

[3] N. Ben Amour. Qualitative Possibilistic Graphical models From Independence to propagation algorithm. PhD thesis, Universitéd'Artois, 2002.

[4] K.Boutouhami, F.Khellaf, An approximate possibilistic graphical model for computing optimistic qualitative decision. In International Conference on Artificial Intelligence and Applications (AIFU2015), Dubai.Vol. 5, 2015, No. 33, pp. 183-1960

[5] Dubois, D.I Fargier, H.I Perny, P.: Qualitative decision theory with preference relations and comparative uncertainty: An axiomatic approach. Artif. Intell., Vol. 148, 2003, No. 1-2, pp. 219-260.

[6] D. Dubois, J. Lang, and H. Prade. Possibilistic logic. In Handbook of Logic in Articial Intelligence and Logic Programming, (D. Gabbay et al., eds, 3, Oxford University Press :pages 439-513, 1994.

[7] D. Dubois, D. Le Berre, H. Prade, and R. Sabaddin. Using possibilistic logic for modeling qualitative decision: Atms based algorithms. In FundamentaInformaticae, 37 :1-30, 1999.

[8] D. Dubois and H. Prade. (with the collaboration of H. Farreny, R. Martin-Clouaire and C. Testemale). Possibility Theory- An Approach to Computerized Processing of Uncertainty. Plenum Press, New York., 1988

[9] D. Dubois and H. Prade. Possibility theory and data fusion in poorly informed environments. In Control Engineering Practice, volume 2(5), pages 811-823, 1994.

[10] D. Dubois and H. Prade. Possibility theory as a basis for qualitative decision theory. In 14th International Joint Conference on Artificial Intelligence (IJCAI'95), Montréal, pages 1924-1930, 1995

[11] D. Dubois and H. Prade. Possibility theory: qualitative and quantitative aspects. In Handbook of Defeasible Reasoning and Uncertainty Management Systems. (D. Gabbay, Ph. Smets, eds.), Vol. 1: Quantified Representations of Uncertainty and Imprecision, (Ph. Smets, ed.) Kluwer, Dordrecht: 169226, 1998.

[12] F. Haned-Khellaf S. Benferhat and I. Zeddigha. Computing optimal optimistic decisions using minbased possibilistic networks. In North American Fuzzy Information Processing Society, Berkeley NAFIPS 2012, JUIN 2012.

[13] L. Garcia and R. Sabbadin. Diagrammes d'influence possibilistes. Revue d'Intelligence Artificielle, 21(4): 521-554, 2007. 
International Journal of Artificial Intelligence \& Applications (IJAIA) Vol. 6, No. 2, March 2015

[14] J. Gebhardt and R. Kruse. Background and perspectives of possibilistic graphical models. In 4th European Conference on Symbolic and Quantitative Approaches to Reasoning and Uncertainty (ECSQARU'97), LNAI 2143, pages 108-121, 1997.

[15] A. Mokhtari S. Benferhat, F. Haned-Khellaf and I. Zeddigha. A possibilistic graphical model for handling decision problems under uncertainty. In 8th conference of the European Society for Fuzzy Logic and Technology, EUSFLAT-2013, September 2013.

[16] R. Sabbadin. Une approche logique de la résolution de problèmes de décision sous incertitude basée sur les atms. In Actes du 11ème Congrés Reconnaissance des Formes et Intelligence Artificielle (RFIA'98), Clermont-Ferrand, pages 391- 400, 20-22 janvier 1998.

[17] G. Shafer. A mathematical theory of evidence. Princeton Univ.Press Princeton, VJ,1976.

[18] P.P Shenoy. Valuation based systems: A framework for managing uncertainty in expert systems. In Fuzzy Logic for the Management of Uncertainty, pages 83104. L. A. Zadeh and J. Kacprzyk, Eds. John Wiley and Sons, New York, NY, 1992.

[19] P.P Shenoy. A comparison of graphical techniques for decision analysis. In European Journal of Operational Research, volume 78, pages 1-21, 1994.

[20] Smirnov, A. Pashkin, M. Chilov, N.I Levashova, T.: KSNet-Approach to knowledge Fusion from Distributed Sources. Computing And Informatics, Vol. 22, 2003, No. 2, pp. 105-142.

[21] Sniedovich, M.: Dynamic Programming: Foundations and Principles, 2nd ed. Pure and Applied Mathematics, CRC Press, Hoboken, 2010.

[22] F.Titouna. Fusion de réseaux causaux possibilistes. $\mathrm{PhD}$ thesis, Université d'Artois, 2009.

[23] L.Zadeh, Fuzzy sets, Information and Control 8, pages 338353, 1965.

[24] L. Zadeh. Fuzzy sets as a basis for a theory of possibility. Fuzzy Sets and Systems, 1:3-28, 1978. 